\title{
EINSTEIN METRICS AND FIBRED RIEMANNIAN STRUCTURES
}

\author{
By TADAyUki Matsuzawa
}

\section{§ 0 . Introduction.}

As is well known, there are many homogeneous Einstein spaces having fibred structures, for example, the total spaces of Hopf fibrations $S^{2 n+1} \rightarrow \boldsymbol{C} P^{n}$, $S^{4 n+3} \rightarrow \boldsymbol{H} P^{n}$ and the complex projective spaces $\boldsymbol{C} P^{2 n+1} \rightarrow \boldsymbol{H} P^{n}$, where the base spaces and the fibres of these fibrations are also Einsteinian. Jenzen [3] and Ziller [5] constructed non-canonical homogeneous Einstein metrics on these standard homogeneous Einstein spaces by varing metrics homothetically along fibre directions. Following Berard-Bergery and Bourguignon [1], this type of metric variation will be called a canonical variation (See $\S 3$, for the canonical variation). Then, in this paper, we describe how a new Einstein metric can be found by the canonical variation in a fibred Riemannian space, whose total space is Einsteinian and not necessarilly homogeneous, and obtain the following theorem which will be proved in $\S 4$.

TheOREM. Let $\pi: M \rightarrow B$ be a fibred Riemannian structure with totally geodesic fibres where the total space is complete. Suppose the total space, base space and fibres are Einstemian. Then the canonical variation at the parameter value equal to

$$
\frac{s^{F}}{f} /\left(\frac{s^{B}}{b}-\frac{s^{F}}{f}\right)
$$

gives another Einstem metric to the total space of the parameter value is positive not one, where $b$ and $f$ are respectively dimension of the base space $B$ and the fibres, $s^{B}$ and $s^{F}$ respectively the scalar curvature of the base space and the fibres.

The author wishes to thank Professor S. Ishihara and Dr. K. Sakamoto for their encouragements and helpful suggestions.

\section{$\S 1$. Structure tensors of a fibred Riemannian structure.}

Let $\pi: M \rightarrow B$ be a fibred Riemannian structure with totally geodesic fibres. We denote by $\langle$,$\rangle the metric of the total space and by \nabla$ the Levi-Civita con-

Received January 12, 1983 
nection determined by the metric $\langle$,$\rangle . Following O'Neill [4], we define in M$ the tensors $T$ and $A$ for arbitrary vector fields $E$ and $F$ by

$$
T_{E} F=\mathscr{C} \nabla_{\mathcal{V}} \mathscr{H} F+\mathscr{H}_{\nabla_{E}} \mathcal{C V} F
$$

and

$$
A_{E} F=\mathcal{C V} \nabla_{\mathscr{H} E} \mathscr{H} F+\mathscr{H}_{\mathscr{K}_{E}} \mathcal{V} F
$$

where $C V E$ and $\mathscr{K} E$ denote the vertical and horizontal parts of vector fields $E$ respectively. The tensor $T$ vanishes since fibres are totally geodesic submanifolds. The tensor $A$ is called the structure tensor and has the properties stated in the following lemmas :

Lemma 1.1. The tensor $A_{E}$ is skew-symmetric operator on the tangent space of $M$ reversing vertical and horizontal subspaces.

LeMMA 1.2. The tensor $A_{E}$ is horizontal i.e.

$$
A_{E}=A_{\mathscr{K} E}
$$

LEMMa 1.3. The structure tensor $A$ is alternative for horizontal vector fields $X$ and $Y$ i.e.

$$
A_{X} Y=-A_{Y} X=\frac{1}{2} \mathcal{V}[X, Y] \text {. }
$$

Proofs of these lemmas are, for example, found in O'Neill [4].

By Hermann [2], completeness of the total space implies that the fibres are isometric each other, then, in the fibred Riemannian structures the Ricci tensor and the scalar curvature of the fibres make senses.

\section{$\S 2$. Einstein condition and the structure tensor.}

We denote by Ric the Ricci tensor of the Riemannian space $(M,\langle\rangle$,$) . Let$ $\left\{Z_{1}, \cdots, Z_{b}\right\}$, where $b=\operatorname{dim} B$, be a local orthonormal basic vector fields in $M$ i. e. unique horizontal vector fields projected to a local orthonormal frame of the base space $B$ by $\pi_{*}$. Then, we have

Proposition 2.1. For vertical vectors $U, V$ and horizontal ones $X$ and $Y$,

$$
\begin{aligned}
& \operatorname{Ric}_{U V}=\operatorname{Ric}_{U V}^{F}+\sum_{i=1}^{b}\left\langle A_{Z_{i}} U, A_{Z_{i}} U\right\rangle, \\
& \operatorname{Ric}_{U X}=-\sum_{i=1}^{b}\left\langle\left(\nabla_{Z_{i}} A\right)_{X} Z_{\imath}, U\right\rangle, \\
& \operatorname{Ric}_{Y Y}=\operatorname{Ric}_{X Y}^{R}-2 \sum_{i=1}^{b}\left\langle A_{Z_{i}} X, A_{Z_{i}} Y\right\rangle,
\end{aligned}
$$


where $\mathrm{Ric}^{F}$ and $\mathrm{Ric}^{B}$ denote the Ricct tensor of the fibres and the base space respectively.

Proof. Let $\left\{U_{1}, \cdots, U_{f}\right\}$ be a local orthonormal vertical vector fields in $M$, where $f$ is the fibre dimension of the fibre. Then, by O'Neill's formula of curvature tensor, we obtain the first and the second identities and

$$
\operatorname{Ric}_{X Y}=\operatorname{Ric}_{X Y}^{B}-3 \sum_{i=1}^{b}\left\langle A_{Z_{i}} X, A_{Z_{i}} Y\right\rangle+\sum_{\jmath=1}^{f}\left\langle A_{X} U_{\jmath}, A_{Y} U_{\jmath}\right\rangle .
$$

But, using next computations, we can rewrite this into the form stated in the proposition: we have, by Lemmas $1.1,1.2$ and 1.3 ,

$$
\begin{aligned}
\sum_{\jmath=1}^{f}\langle & \left.A_{X} U_{\jmath}, A_{Y} U_{\jmath}\right\rangle \\
& =\sum_{\jmath=1}^{f} \sum_{\imath=1}^{b}\left\langle A_{X} U_{\jmath}, Z_{\imath}\right\rangle\left\langle A_{Y} U_{\jmath}, Z_{\imath}\right\rangle=-\sum_{\jmath=1}^{f} \sum_{\imath=1}^{b}\left\langle U_{\jmath}, A_{X} Z_{\imath}\right\rangle\left\langle A_{Y} U_{\jmath}, Z_{\imath}\right\rangle \\
& =-\sum_{\jmath=1}^{f} \sum_{\imath=1}^{b}\left\langle A_{Y} A_{X} Z_{\imath}, Z_{\imath}\right\rangle=\sum_{\imath=1}^{b}\left\langle A_{X} Z_{\imath}, A_{Y} Z_{\imath}\right\rangle \\
& =\sum_{\imath=1}^{b}\left\langle A_{Z_{i}} X, A_{Z_{i}} Y\right\rangle
\end{aligned}
$$

COROLlary 2.2. If $M, B$ and the fibres are Einsteman, then we obtam,

$$
\begin{aligned}
\sum_{\imath=1}^{b} & \left\langle A_{Z_{i}} U, A_{Z_{\imath}} V\right\rangle=\left(\frac{s^{M}}{f+b}-\frac{s^{F}}{f}\right)\langle U, V\rangle, \\
& -\sum_{\imath=1}^{b}\left\langle\left(\nabla_{Z_{i}} A\right)_{X} Z_{\imath}, U\right\rangle=0, \\
& -2 \sum_{\imath=1}^{b}\left\langle A_{Z_{i}} X, A_{Z_{\imath}} Y\right\rangle=\left(\frac{s^{M}}{f+b}-\frac{s^{B}}{b}\right)\langle X, Y\rangle .
\end{aligned}
$$

Proof. Since the assumptions imply that

$$
\begin{aligned}
\operatorname{Ric} & =\frac{s^{M}}{f+b}\langle,\rangle, \\
\operatorname{Ric}^{F} & =\frac{s^{F}}{f}\langle,\rangle, \\
\operatorname{Ric}^{B} & =\frac{s^{B}}{b}\langle,\rangle,
\end{aligned}
$$

Proposition 2, 1 implies directly this corollary.

Q.E.D. 


\section{§3. Canonical variation.}

We define new metrics $\langle,\rangle_{t}$ on the total space with positive parameter $t$

$$
\langle E, F\rangle_{t}=t\langle\mathcal{V} E, U F\rangle+\langle\mathscr{H} E, \mathscr{H} F\rangle,
$$

for arbitrary vectors $E$ and $F$.

Lemma 3.1. The Levi-Civita connection $\nabla^{t}$ determined by the metruc $\langle,\rangle_{t}$ is given by the formulas

$$
\begin{aligned}
& \nabla_{U}^{t} V=\nabla_{U} V, \\
& \nabla_{U}^{t} X=\nabla_{U} X+(t-1) A_{X} U, \\
& \nabla_{X}^{t} V=\nabla_{X} V+(t-1) A_{X} V, \\
& \nabla_{X}^{t} Y=\nabla_{X} Y,
\end{aligned}
$$

for vertical vector fields $U, V$ and horizontal vector fields $X, Y$. Then, the structure tensor $A^{t}$ of the new fibred Riemannan structure is given by

$$
A_{X}^{t} V=t A_{X} V, \quad A_{X}^{t} Y=A_{X} Y .
$$

Proof. The first and fourth formulas are trivial. To prove the second formula, we need the following computations:

$$
\begin{aligned}
2\left\langle\nabla_{U}^{t} X, Y\right\rangle & =2\left\langle\nabla_{U}^{t} X, Y\right\rangle_{t} \\
& =U\langle X, Y\rangle_{t}+X\langle U, Y\rangle_{t}-Y\langle U, X\rangle_{t} \\
& +\langle[U, X], Y\rangle_{t}+\langle[Y, U] X\rangle_{t}+\langle U,[X, Y]\rangle_{t} \\
= & U\langle X, Y\rangle+\langle[U, X], Y\rangle+\langle[Y, U], X\rangle \\
& +\langle U,[Y, X]\rangle+(t-1)\langle U,[Y, X]\rangle \\
= & 2\left\langle\nabla_{U} X, Y\right\rangle+2(t-1)\left\langle U,-A_{X} Y\right\rangle \\
= & 2\left\langle\nabla_{U} X+(t-1) A_{X} U, Y\right\rangle .
\end{aligned}
$$

Then we have

$$
\mathscr{H} \nabla_{U}^{t} X=\mathscr{H} \nabla_{U} X+(t-1) A_{X} U
$$

Having

$$
c \nu \nabla_{U}^{t} X=c V \nabla_{U} X
$$

by similar computation, we obtain the second formula. The third formula is obtained by the same way as the second. Q.E.D. 
LEMMA 3.1 implies that the fibration $\pi:\left(M,\langle,\rangle_{t}\right) \rightarrow B$ gives a fibred Riemannian structure with totally geodesic fibres.

LEMmA 3.2. Let $\mathrm{Ric}^{t}$ denote the Ricci tensor of the Riemannian space $\left(M,\langle,\rangle_{t}\right)$. Then,

$$
\operatorname{Ric}_{U X}^{t}=t \operatorname{Ric}_{U X}
$$

Thus $\operatorname{Ric}_{U X}^{t}$ vanushes whenever $\operatorname{Ric}_{U X}$ vanishes.

Proof. Lemma 1.2 and 3.1 imply

$$
\mathscr{V}\left(\nabla_{Z_{\imath}}^{t} A^{t}\right)_{X} Z_{\imath}=\mathscr{Q}\left(\nabla_{Z_{\imath}} A\right)_{X} Z_{\imath},
$$

so the second formula of Proposition 2.1 and the definition of $\langle,\rangle_{t}$ imply the lemma.

Q.E.D.

\section{$\S 4$. Proof of Theorem.}

By Proposition 2.1, Corollary 2.2 and Lemmas 3.1, 3.2, we have

$$
\begin{aligned}
\operatorname{Ric}_{U V}^{t} & =\frac{s^{F}}{f}\langle U, V\rangle+\sum_{\imath=1}^{b}\left\langle A_{Z_{i}}^{t} U, A_{Z_{\imath}}^{t} V\right\rangle_{t} \\
& =\frac{s^{F}}{t f}\langle U, V\rangle_{t}+t^{2} \sum_{\imath=1}^{b}\left\langle A_{Z_{i}} U, A_{Z_{\imath}} V\right\rangle \\
& =\left(\frac{s^{F}}{t f}+t\left(\frac{s^{M}}{f+b}-\frac{s^{F}}{f}\right)\right)\langle U, V\rangle_{t},
\end{aligned}
$$

$\operatorname{Ric}_{U X}^{t}=t \operatorname{Ric}_{U X}=0$,

$$
\begin{aligned}
\operatorname{Ric}_{X Y}^{t} & =\frac{s^{B}}{b}\langle X, Y\rangle-2 \sum_{i=1}^{b}\left\langle A_{Z_{i}}^{t} X, A_{Z_{i}}^{t} Y\right\rangle_{t} \\
& =\frac{s^{B}}{b}\langle X, Y\rangle_{t}-2 t \sum_{i=1}^{b}\left\langle A_{Z_{i}} X, A_{Z_{i}} Y\right\rangle \\
& =\left(\frac{s^{B}}{b}+t\left(\frac{s^{M}}{f+b}-\frac{s^{B}}{b}\right)\right)\langle X, Y\rangle_{t} .
\end{aligned}
$$

Consequently, if

$$
\frac{s^{F}}{t f}+t\left(\frac{s^{M}}{f+b}-\frac{s^{F}}{f}\right)=\frac{s^{B}}{b}+t\left(\frac{s^{M}}{f+b}-\frac{s^{B}}{b}\right),
$$

then $\langle,\rangle_{t}$ gives Einstein metric. By solving this equation of $t$, we have two solutions, i.e.

$$
t=1, \quad t=\frac{s^{F}}{f} /\left(\frac{s^{B}}{b}-\frac{s^{F}}{f}\right) .
$$


Therefore the theorem is proved.

Q.E.D.

\section{$\S 5$. Examples.}

For the Hopf fibring $S^{4 n+3} \rightarrow \boldsymbol{H} P^{n}$, the fibres are isometric to the ordinary sphere $S^{3}$ and

$$
\frac{s^{F}}{f}=2, \quad \frac{s^{B}}{b}=4(n+2) .
$$

Then

$$
\frac{s^{F}}{f} /\left(\frac{s^{B}}{b}-\frac{s^{F}}{f}\right)=\frac{1}{2 n+3} .
$$

This is Jenzen's example [3]. For the fibration $\boldsymbol{C} P^{2 n+1} \rightarrow \boldsymbol{H} P^{n}$, the fibres are isometric to the sphere $S^{2}(4)$ with constant sectional curvature 4 and

$$
\frac{s^{F}}{f}=4, \quad \frac{s^{B}}{b}=4(n+2) .
$$

Then

$$
\frac{s^{F}}{f} /\left(\frac{s^{B}}{b}-\frac{s^{F}}{f}\right)=\frac{1}{n+1}
$$

This is Ziller's example [5].

\section{REFERENCES}

[1] Berard Bergery, L. and Bourguignon, J.P., Laplacian and Riemannian submersions with totally geodesic fibres, Lecture Notes in Math. 838 30-35 (1981).

[2] Hermann, R., A sufficient condition that a mapping of Riemannian manifolds be a fibre bundle, Proc. Amer. Math. Soc. 11 236-242 (1960).

[3] Jenzen, G. R., Einstein metrics on principal fibre bundles, J. Diff. Geom. 8 599-614 (1973).

[4] O'Neill, B., The fundamental equations of a submersion, Michigan Math. J. 13 459-469 (1966).

[5] Ziller, W., Homogeneous Einstein metrics on spheres and projective spaces, Math. Ann. 259 351-358 (1982).

TOKyo Institute of TECHNOLOgy 